flow of vitiated blood to their mucous lining. The congestions which arise in other parts, in consequence of a direct infection of the blood, may terminate in the effusion of serum, lymph, pus, or blood, either separately or mixed together in various proportions. In some rare instances, the secondary inflammation may terminate in resolution; of this the following case affords an instructive example.

CASE XIV. ${ }^{1}$ G. B., æt. 31, was admitted into St. George's Hospital for an aneurism in the left groin, for which the external iliac artery was tied. On the evening after the operation, the whole abdomen became painful and tender; the pulse was frequent, the skin hot, and the tongue dry and brown. The patient was bled, and on the following day the bleeding was repeated. The adhesions of the edges of the wound were destroyed, and gave exit to some sanious matter contained within. On the third day, none of the local symptoms were relieved, and the constitutional symptoms were much aggravated. The right shoulder now became affected with intense pain, accompanied by swelling and tenderness. Half a pint of wine was prescribed to be taken daily, with such nourishment as the stomach could receive. The pain in the shoulder, with some degree of swelling, continued for some time; but on the quantity of the stimulus being farther increased, this, as well as the other symptoms, gradually subsided.

This case is interesting, not only as showing that a secondary inflammation may terminate in resolution, but also as indicating the kind of constitution in which such secondary affections are likely to occur. Three months after the patient was discharged from the hospital as cured, the pulsation returned in the tumour, which again enlarged to a very considerable size. It is therefore evident that the coagulating power of his blood had from some cause been greatly impaired : for had this not been the case, the coagula which had been allowed such ample time to form, would have permanently closed the dilated vessel.

13, Dover Street, Piocadilly, June 1851.

\title{
HYSTERICAL AFFECTIONS OF THE HIP JOINT.
}

By WILLIAM COULSON, Esq., F.R.S.C.Eng., Surgeon to St. Mary's Hospital ; and to the Magdulen Hospital, etc.

I was consulted in the case of a young lady, nineteen years of age, pale and delicate looking, of highly nervous and excitable temperament, on account of an affection of the right hip joint, for which she had been under surgical treatment, and for a considerable time confined to the recumbent position. The frequent application of leeches, and the employment of counter-irritation in every possible form, had proved without avail ; she lay helpless and bed-ridden, complaining of severe pain extending over the whole lower extremity, and impressed with a firm conviction that she would remain a cripple for life. She shrunk from the slightest attempt to examine the limb, declaring that the pain she experienced was insupportable. My attention, however, was

1 Related by Sir Benjamin C. Brodie in the Medical Gazette. 
directed to the fact, that her general health had not ouffered to an extent corresponding with what might have been anticipated from the severity of the local suffering, and the duration of her illness and confinement. The head of the femur was evidently in the acetabulum; there were no evidences of thickening or of disorganisation of the structures composing the joints; the pain was diffused, and her exaggerated complaints seemed as much excited when the integuments were lightly touched, as when firm pressure was made over the bones. No persuasions of mine could induce this patient to leave her bed, or to use the limb. I ordered her different preparations of steel, quinine, and other suitable remedies, but there was no perceptible change for the better. She was lying in this helpless state, when intimation was sent her that her father had been suddenly taken ill, and was not expected to live. She was conveyed in an invalid carriage to a distant part of the country, where the family resided, and arrived in time to see her father expire. By this loss, the means of living became much reduced; the family, which was large, could no longer pretend to their former state of affluence, and the different members were called upon, for the first time, to exert themselves to meet their calamity. This young lady upon being made acquainted with the particulars-for misfortune it was none to her-one day suddenly rose from her couch and walked. She exerted herself much, both mentally and bodily, for the good of those around her, seemed to forget her long illness, and has ever since remained well. That the joint was not affected with any organic change is clear from the result of the case. The disease was one of those obscure hysterical affections, more common among the rich than the poor, during which the impression of pain is in a morbidly excited and perverted state, and the power of the will over a certain group of muscles is suspended.

A limb was removed by a surgeon from a patient, whose symptoms, in many respects, corresponded with those in the case $I$ have above related. The girl was in humble life, but well brought up, and was much noticed by some ladies who superintended the school where she was educated. She was of pale complexion, with dark hair and eyes, and of highly excitable temperament. Without apparent cause, severe pain came on in the right knee; the leg became flexed upon the thigh, and the slightest effort at extension excited impressions of intense suffering. She became an interesting object of pity; numerous professional opinions were asked by her patronesses, and frequent visits were paid to her by different surgeons. All, however, urged the necessity of her leaving her bed and using the limb; a measure to which she professed herself unequal. At the expiration of three years, during which her condition varied but slightly, a surgeon was induced, upon her representations, to recommend the amputation of the limb. To this she assented; the operation was performed, and the wound healed without any unfavourable symptom. Upon examination, the joint was found to be perfectly healthy. The synovial membrane retained its normal delicacy and transparency; the articular cartilage was slightly thinned, as is usually the case in a limb which has been long disused; the bones were light and easily sawn, but otherwise healthy; and there were no morbid changes in any of the surrounding structures.

It has been calculated, that at least four-fifths of the females among 
the higher classes of society, supposed to be suffering from diseases of joints, are, in truth, affected with hysteria, and with nothing else. As, in the one case, absolute rest is requisite to effect a cure; and in the other, the patient must be urged, nay, even forced to take exercise, in spite of complaints of pain, it is important that the surgeon should be familiar with everything that will assist him to form an early and accurate diagnosis.

In hysterical affections of the hip joint, the patient from the first complains of pain in the part, and not in the knee, as is frequently the case when organic changes are commencing in the joint itself. The pain which the patient describes as most severe is not limited to one spot, but extends over the buttock to the lumbar region, and down the thigh. It is this general diffusion of pain which constitutes one of our most useful distinctions between this affection and disease of the hip joint. From the commencement, the patient complains of such aggravation of pain by pressure and motion, that she confines herself to one position of the limb; and yet upon occasions, when the mind is otherwise occupied, or during sleep, she will move it without complaint. The sensibility of the limb to the touch is frequently so great, that the slightest pressure on any part of the hip or thigh will cause the patient to scream; she shrinks involuntarily from the mere approach of the hand, but nevertheless, upon a careful examination, these morbid conditions will be found to exist more in the skin than in the deep-seated parts. As Sir Benjamin Brodie, who first directed attention to these important affections, observes: "If you pinch the skin, lifting it at the same time off the subjacent parts, the patient complains more than when you forcibly squeeze the head of the thigh bone into the sockct of the acetabulum." "The more the patient's attention is directed to the part, the more the pain is increased; but if her attention be directed otherwise, she will hardly complain, and the pain does not interfere with her rest. In disease involving the structures of the joint, there is nothing of which the patient complains so much as the inability to sleep at night. Just as "Nature's soft nurse" comes to soothe the suffering and exhausted frame, a sudden start, or pain, or cramp, banishes sleep, and the pafient dreads again to close her eyes. In hysterical affections, on the contrary, the sleep is calm and refreshing; and this, pcrhaps, is a great reason why the general health so slightly suffers, even in long-protracted cases. The pain is anomalous in its character, and different from the result of inflammation, or other organic changes. It may not be always easy to arrive at the truth, for such is the morbid constitution of the mind, that when leading questions have on a previous occasion been put, the patient will, as it were, take the hint, and symptoms, for which we previously may have in vain inquired into, will now actually present themselves. As for the motives for such deception, "to become an interesting object", says Dr. Prout, " " is an innate and characteristic feeling of the human mind; and if in early life this feeling in its natural and legitimate form has been thwarted or disappointed, it is almost sure to deviate into some other channel; and the ruling passion is displayed in attempt.

I Brodr, Sir R. G. Toctures on Incal Nervots Jiceases, p. 88.

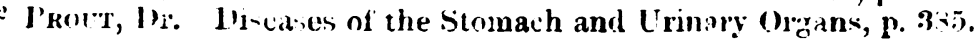


ing to excite pity and commiseration, when she cannot excite erotic sentiment."

In some instances, there is no visible alteration about the joint or neighbouring parts of the limb; in others, there is general puffiness and tumefaction of the whole hip. The swelling, however, is rarely considerable, and depends on the effusion of fluid into the surrounding areolar tissue. It corresponds with what may be observed in some other neuralgic cases; thus Sir Benjamin Brodie relates a case of facial tic douloureux, attended with severe pain and swelling of the face, although there could be no doubt that the seat of the disease was not in the nerves, but in the brain itself. I have seen similar cases. In one, severe paroxysms of pain, attended with considerable œdema over those parts of the face supplied by the first and second divisions of the fifth, was the result of some obscure disease of the brain, in a man otherwise healthy, of temperate habits, and of powerful frame. When the tumefaction is limited to the immediate neighbourhood of the hip joint, it increases the difficulty of distinguishing this affection from organic disease, more especially as, in some forms of scrofula, the muscular excitement of a subacute character, dependent possibly upon irritation caused by the deposit of tubercle, spreads to the neighbouring parts, producing an undefined fulness and puffiness. In these cases, however, the swelling eventually is more defined, and proceeds from the formation of matter within the joint ; in hysteria, the interior of the joint is quite sound.

I have often witnessed the periodical change of temperature mentioned by Sir Benjamin Brodie (Op. cit. p. 284), affecting cither the joint, or even the whole limb. In the morning it is cold, pale, and shrunk; towards evening there is evidence of a more active circulation, and the surface of the skin is evidently red, hot, and shining. During the night, the heat and redness subside ; and these alternations are as regular as the paroxysms of an ague. A patient who was some time under my care, was extremely susceptible of changes of temperature in the part; the knee, the part affected, was usually colder than the opposite joint; but towards evening it became warm, and quite damp with perspiration.

In all these cases, there is a great tendency to muscular contraction, and the limb is thrown into that position which it would assume after the influence of the will is removed, as in sleep. The thigh is bent upon the pelvis, the leg is bent upon the thigh; and, unless means be taken from the commencement to counteract this deformity, it gives the surgeon more trouble than the original complaint. The muscles undergo a sort of atrophy, and become paler than natural ; the tendons contract cellular adhesions with parts around, so that very considerable and continued force may be requisite to free the limb, and to bring it to its proper bearings. I have known it necessary for the surgeon to perform the operation of the subcutaneous division of tendons, in the case of a contracted limb in an hysterical girl. Both the outer and the inner ham-string tendons were divided, when the leg was readily extended, and the patient recovered the use of the limb. The muscles about the hip, arising from the pelvis, do in some cases contract in such a manner, as to prevent the patient putting the heel to the ground on a level with that of the sound leg; and at times the limb is even so 
much drawn out of its natural position, as to simulate dislocation of the femur upon the ilium.

A young lady, the subject of hysterical hip disease, came under my care, the case having previously been mistaken for disease of the joint, and moxæ applied. The knee was bent; the limb inverted; the thigh rested upon its fellow, and the head of the bone was preternaturally prominent. A superficial observer might have easily mistaken it for dislocation. The most positive assurance on my part could scarcely remove the impression which existed in the minds of the parents that there was no organic disease in the joint, and that there was no necessity to pursue the plan of counter-irritation and confinement. My patient was first directed to take out-door exercise in a carriage; with much trouble the limb was at length straightened; then she was persuaded to attempt to walk; and by these means she ultimately recovered.

Sir Charles Bell relates a case of this kind ; and similar cases are not uncommon, in which " the knee was bent almost to the bursting of the ligaments, and the foot turned in so extraordinary a manner that the great toe lay close to the anus."

The age most liable to this affection is from fifteen to twenty; but when once developed, it may continue for a long period. I have met with cases in which the catamenia were irregular or scanty; but in other instances they have not deviated from health. The bowels are sometimes, but by no means invariably, constipated.

I will now sum up the symptoms by which this affection is distinguished from disease of the hip joint. In the nervous affection, pain is felt, from the commencement, in the hip, and extends to the loins and down the thigh; there is great general nervous excitability, and extreme sensitiveness in the part; and the patient is from the first unable to walk. Combined with this extreme amount of expressed suffering, the trochanter major retains its proper bearing to the spine of the ileum. There is not the characteristic wasting of the glutei muscles, and consequently no flattened appearance of the nates. Pressure in these situations, where the bone approaches almost the surface, does not excite greater pain than in the other parts. There are none of those involuntary startings during sleep, which cause the patient such intense agony. On the contrary, however loud may be the complaints of the painful condition of the limb while the patient is awake, no sooner is she asleep, than she rests well and calmly throughout the night. In true hip disease, the reverse is the case; for, when nature is exhausted by suffering, and the patient faint from want of rest, the sleep, if unaided by the administration of opium, is broken by sudden cramps, shooting pains, and frightful dreams, or vague anticipations of coming pain.

There is one circumstance attending this affection of the joint, which is calculated to mislead the inexperienced practitioner. I allude to its being often described as consecutive to a blow or other injury. In fact, many surgeons believe that there is generally some such cause, which determines the manifestations of these nervous phenomena in any one particular joint. I cannot say that I share largely in this view. Were local injuries alone the exciting cause, the pain would 
be equally felt in such constitutions, in whatever joint were the seat of the accident; whereas, it is well known that, in by far the greater number of cases, the neuralgia is in the hip or knee.

As regards the pathology of these affections, we know little. The joint, the seat of pain, offers no abnormal deviations. It may, however, be asked, is not this affection seated in the spinal cord, or in the brain? Can we explain the phenomena of pain and muscular contraction, upon the supposition that the spinal cord, to which all motor and sensitive nerves are attached, is in a morbidly excited state, and that the brain responds truly to the impressions which it receives? $O r$ is it that the functions of the brain are all perverted; and that an inquiry must be conducted upon the same principle as if we were seeking to investigate the reason why, as in the insane, objects are seen which do not exist, and sounds are heard which were never uttered? From the wonderfully sudden change which a strong mental impression can effect in one who is the subject of an hysterical affection of a joint, I am inclined to believe that it is to the sensorium we must mainly look as the organ morbidly affected; although I am fully aware that, in many instances, there are evident proofs of a highly sensitive and excited condition of the cord.

A young lady, nineteen years of age, complained of a constant gnawing pain in the knee joint ; of stiffness and weakness, from which she feared a sudden fall. Her parents wished the joint to be strapped and bandaged; but feeling convinced that the disease was of a nervous character, this plan was not adopted. At the time of each menstrual period, the pain was more severe; and when seated at work, or engaged in reading, she was subject to a violent convulsion, without increase of pain, and incontrollable by the will, affecting the muscles of the whole body. It was a strongly marked instance of reflex action; the convulsion being so strong, that she would rise from her seat and forcibly grasp whatever object she was holding. The same phenomena would occur, if, at these periods, she was suddenly exposed to any risk of receiving a blow on the affected knee; the movement then being equally general and involuntary. There was, however, no increased pain; the sensation which the brain appreciated remaining unchanged, however violent the spasm of the leg.

"The existence," says Dr. Conolly, "of an original susceptibility in excess, in the nervous system of hysterical patients, is an assumption warranted, we conceive, by all observation. The natural or congenital constitution of the nervous system, and even of different portions of it, is most plainly discerned to be different in different individuals. From the very cradle may be observed a different degree of sensibility to impressions; and even a different countenance, impressed by the hand of nature herself, before human feelings have written their deeper lines upon it." "In the hysterical patient, we may observe the most intense development of susceptibility, connected with a singular proneness to irregular action, often arising from slight causes; the natural proportion designed to exist between the impressions of the external world, and the sensient human system, being in them not preserved."1

I Conowry, Dr.J. Cyclopædia of Practical Medicine, Art., HysterLa : vol.ii, p. 576. 
With regard to the treatment of this affection, it is obvious that, if it be mistaken for organic disease of the hip joint, the surgeon will be led to adopt a line of practice, not merely useless, but positively injurious. The seat of the disease being in the nervous centre, little can be done locally, with any benefit or relief to the patient. We are, therefore, called upon to direct our remedies to the state of the nervous system generally. The patient must be persuaded to leave her couch as soon as possible. It is useless to wait until the painful sensations have subsided. She must take carriage exercise daily, be in the open air as much as possible, and in the society of lively and cheerful companions of her own age. The diet should be plain and nutritious. In the common forms of hysteria, I find the vegetable tonics more efficacious than the metallic; but, in that now under consideration, they possess no superiority. Their employment, however, is by no means to be laid aside; for in all cases where the tongue is foul, and there are other evident signs of derangement of the digestive organs, they are more beneficial than any preparations of the metals. But under different circumstances, as when the appetite is tolerably good, the tongue clean, and the bowels regular, then it is that the metallic tonics may be tried with great effect. In most neuralgic affections, the preparations of iron prove successful; and among these the carbonate stands justly preeminent. It may be prescribed in as large a dose as the stomach will bear without inconvenience. Among the medicines that directly soothe the pain, none are preferable to valerian; for though much less powerful than opium and belladonna, it neither constipates the bowels, nor enfeebles the system.

"The medicine which I have found the most useful," says Dr. Copland, " is the spirits of turpentine, prescribed in various modes, internally and externally, and administered in enemata; the preparations of iodine, alone, or with narcotics and camphor. These, however, should be associated with suitable adjuvants; amongst which, the several narcotics and antispasmodics are the most important. The warm or vapour bath, simple or variously medicated; mental excitement, and exercise taken regularly and energetically, and employment of the mind, are also important aids in the treatment. The affections of the joints are sometimes accompanied, or even alternated, with severe nervous pains in the extremities, and occasionally with tenderness in some portion of the spine. In such cases the treatment hardly requires any material alteration. In those which have come under my care, I have very frequently prescribed the spirit of turpentine, as already stated, and often repeatedly in enemata; and after two or three doses of it, I have commenced with the preparations of iodine, conjoined with henbane, opium, or belladonna. Whilst the iodine has been given, the turpentine has been administered in enemata, from time to time; and embrocations or liniments assiduously employed."

When the pain has been extreme and almost insufferable, the local abstraction of blood by leeches has afforded relief; but it has been only temporary: and, as the effect of the loss of blood necessarily is to weaken the frame, their application cannot be often repeated without producing permanent mischief.

Counter-irritants, such as blisters, setons, stimulating liniments, 
mustard-poultices, and the like, all aggravate instead of relieving the pain. The belladonna or opium plaster, and sedative liniments, are the only local applications which I employ.

It may, however, be fairly questioned to what extent medical treatment in these cases is productive of good, unless there exists some evident derangement, against which our remedies may be directed. Far more is to be effected by moral treatment. Those absurd fancies to which young women of the present day are peculiarly susceptible, and which prevent their giving way to the vivacity and buoyancy of spirits suited to their age, should be carefully checked. The mind should be trained to regain its elasticity, instead of brooding over melancholy forebodings, or seeking to excite sympathy and compassion. The patient (cruel as it may seem) should not be allowed the privileges of a sick-room; nor should she be permitted to recline upon the sofa during the day, an interesting object of solicitude to her friends, and of care to a medical attendant. By being compelled to move the limb, and enter into society, patients have been known to recover, after the complaint had resisted every other kind of treatment.

The time of cure varies. Sometimes it is instantaneous; at other times it is slow. The time, however, does arrive, when this morbid state of the nervous system passes away, and the patient begins, though somewhat late in the day, to feel grateful to those to whose judgment and perseverance she owes her recovery. Above all, let it be remembered, that in this affection, the power of motion is present from the beginning; but the will to exercise it lies dormant, until called into action by some sudden emergency, or excited by moral and remedial means.

\section{REMARKS ON THE NATURE AND TREATMENT OF HAY-FEVER.}

By F. W. MACKENZIE, M.D., Physician to the Paddington Free Dispensary for the Diseases of Women and Children, Fellow of University College, etc.

Ir is well known that the respiratory mucous membrane is in some persons peculiarly susceptible to certain impressions, and readily disposed to take on morbid action in consequence. The perfume of particular flowers in one,- the effluvia from ipecacuanha in another, - the emanations from hay and grass in a third,- the smell of certain animals in a fourth,- and the presence of minute quantities of dust in the atmosphere in a fifth, - will give rise to much irritation of this membrane, and in some cases to severe consecutive constitutional disorder. In what this peculiar susceptibility consists, we neither know, nor shall probably ever discover; but the fact itself is undeniable, and in a practical point of view is one of extreme interest.

In the different instances which have been mentioned, it is probable that a similar state of the mucous membrane is induced in each; and that although the causes may differ, their ultimate action is the same; that this is primarily upon the nervous system, or those parts of it which are more immediately connected with the respiratory mucous 